\title{
Effect of Lateral Dimension of Natural Graphite on Fabrication of Three-Dimensional Graphene Aerogels for High-performance Li-metal anodes
}

Huan Zhang ${ }^{1,2}$, Xinxiu Yan $^{3}$, Yingjun Qiao $^{1,2}$, Huimin Shang ${ }^{1,2}$, Meiling Huang ${ }^{1}$, Xuemei Zhou ${ }^{1}$, Hanxiao Zhou ${ }^{1,2}$, Tianhui Li ${ }^{1,2}$, Jingjing Gao ${ }^{1,2}$, Wenjing Liu ${ }^{1,2}$,

Meizhen $Q u^{1}$, Gongchang Peng ${ }^{1, *}$, Xue $\mathrm{Li}^{4, *}$

${ }^{1}$ Chengdu Institute of Organic Chemistry, Chinese Academy of Sciences, Chengdu, Sichuan 610041, China

${ }^{2}$ University of Chinese Academy of Sciences, Beijing 100049, China

${ }^{3}$ School of Chemistry and Environment, Southwest Minzu University, Chengdu 610041, China

${ }^{4}$ National and Local Joint Engineering Laboratory for Lithium-ion Batteries and Materials Preparation Technology, Faculty of Metallurgical and Energy Engineering, Kunming University of Science and Technology, Kunming 650093, PR China

*E-mail: pgc0102@ cioc.ac.cn (Gongchang Peng), 438616074@qq.com (Xue Li))

doi: $10.20964 / 2021.04 .08$

Received: 1 December 2020 / Accepted: 25 January 2021 / Published: 28 February 2021

Lithium metal, regarded as one of the most potential anodes for future battery systems, has attracted much attention for a long time. Nonetheless, uncontrollable Li dendrites growth and almost infinite volume change hinder the reliable implementation of lithium anode. Three-Dimensional graphene aerogel is an ideal lithium metal host material because of its abundant porous structure and large surface area, which can relieve the volume change, as well as reduce the effective current density. The lateral dimension of raw material natural graphite will affect the morphology and hierarchical structures of graphene aerogels, thereby affecting the electrochemical properties as a lithium host material. A series of graphene aerogels were prepared from natural graphite with different lateral dimensions $(3.4 \mu \mathrm{m} 、 11$ $\mu \mathrm{m} 、 44 \mu \mathrm{m} 、 178 \mu \mathrm{m})$ for Li host material. The GA-11/Li composite anode exhibits the best electrochemical performance. The symmetrical cell with GA-11/Li electrode shows stable cycling for $600 \mathrm{~h}$ at $2 \mathrm{~mA} \mathrm{~cm}^{-2}$ for $2 \mathrm{mAh} \mathrm{cm}{ }^{-2}$. Surprisingly, the Li-S full cell with GA-11/Li anode delivers a high initial specific discharge capacity of $9.38 \mathrm{mAh} \mathrm{cm}^{-2}$ and stable cycle stability.

Keywords: Lithium metal anode, graphene aerogel, different lateral dimensions, cycling stability

\section{FULL TEXT}


(C) 2021 The Authors. Published by ESG (www.electrochemsci.org). This article is an open access article distributed under the terms and conditions of the Creative Commons Attribution license (http://creativecommons.org/licenses/by/4.0/). 